\title{
MODIFICAÇÕES QUÍMICAS EM SOLOS ÁCIDOS OCASIONADAS PELO MÉTODO DE APLICAÇÃO DE CORRETIVOS DA ACIDEZ E DE GESSO AGRÍCOLA
}

\author{
Paulo Roberto Ernani ${ }^{1,3 *}$; Michele Schurmann Ribeiro ${ }^{2}$; Cimélio Bayer ${ }^{1}$ \\ ${ }^{1}$ Depto de Solos - UDESC, C.P. 281 - CEP: 88520-000 - Lages, SC. \\ ${ }_{3}^{2}$ Pós-Graduanda em Solos - UDESC. \\ ${ }^{3}$ Bolsista CNPq. \\ *Autor correspondente <prernani@cav.udesc.br>
}

\begin{abstract}
RESUMO: Os benefícios do calcário aplicado sobre a superfície do solo no rendimento vegetal podem estar associados à melhoria na composição química das águas de percolação. Esse trabalho objetivou avaliar alterações na fase sólida e na solução percolada em dois solos ácidos (Latossolo Bruno Álico e Cambissolo Húmico Álico), ocasionadas pelo método de aplicação (na superfície ou incorporado) de corretivos da acidez $\left(\mathrm{CaCO}_{3}\right.$ e $\left.\mathrm{MgCO}_{3}\right)$ e de gesso agrícola. $\mathrm{O} \mathrm{CaCO}_{3}$ foi utilizado na dose equivalente a 0,25 daquela necessária para elevar o pH a 6,0, e os demais em doses molares equivalentes. $\mathrm{O} \mathrm{CaCO}_{3}$ foi ainda incorporado nas doses de 0; 0,5; 1,0 e 1,5 vez a necessidade de calcário para pH 6,0. Em colunas de lixiviação, com $1,5 \mathrm{~kg}$ de solo, percolou-se água destilada durante 12 semanas num volume de $200 \mathrm{~mL}$ semana $^{-1}$. Os corretivos da acidez, quando incorporados aos solos, aumentaram o pH das duas fases, diminuíram o Al trocável, porém não afetaram o Al percolado; quando foram aplicados na superfície, não afetaram nenhum parâmetro da solução percolada, e alteraram a composição química somente da camada de 0 a $2,0 \mathrm{~cm}$ de profundidade. O gesso agrícola diminuiu o pH do solo e da solução percolada, não afetou o Al trocável, mas percolou muito mais $\mathrm{Ca}, \mathrm{Mg}$ e Al que os corretivos da acidez, principalmente quando foi incorporado ao solo. A mobilidade dos cátions integrantes dos corretivos foi muito pequena, e a aplicação de calcário na superficie dos solos não afetou a composição química da solução coletada $30 \mathrm{~cm}$ abaixo.
\end{abstract}

Palavras-chave: calagem superficial, percolação de íons, cátions trocáveis

\section{CHEMICAL MODIFICATIONS IN ACID SOILS CAUSED BY ADDITION OF GYPSUM OR LIMESTONE}

\begin{abstract}
The increase on crop yield caused by lime addition on the soil surface may be associated to improvements in the chemical composition of the percolating water. This study was carried out to evaluate chemical changes in the solid phase and in the percolating water in two acid soils (Oxisol and Inceptisol) caused by the application method (incorporated or on soil surface) of gypsum, $\mathrm{CaCO}_{3}$, or $\mathrm{MgCO}_{3}$. Calcium carbonate was used at a rate equivalent to 0.25 of that recommended by the SMP method to raise soil water $\mathrm{pH}$ to 6.0; gypsum and $\mathrm{MgCO}_{3}$ were used at the same molarity rate. Additional $\mathrm{CaCO}_{3}$ were $(0,0.50,1.0$ and 1.5 times that recommended by the SMP method) was also incorporated. Treated soil samples $(1.5 \mathrm{~kg})$ were transferred to leaching columns, and distilled water $\left(200 \mathrm{~mL}\right.$ column $^{-1}$ week $\left.^{-1}\right)$ were added on soil surface during twelve weeks. Incorporation of lime $\left(\mathrm{CaCO}_{3}\right.$ and $\left.\mathrm{MgCO}_{3}\right)$ into the soils increased $\mathrm{pH}$ of both phases, decreased exchangeable $\mathrm{Al}$, but had no effect on $\mathrm{Al}$ in the percolated solution. When carbonates were applied on soil surface, they had no effect on the leached solution, and affected the chemical composition of the solid phase only in the top 2.0 centimeters. Addition of gypsum decreased soil and solution $\mathrm{pH}$, had no effect on exchangeable $\mathrm{Al}$, but leached greater amounts of $\mathrm{Ca}, \mathrm{Mg}$ and $\mathrm{Al}$ than limestone, especially when gypsum was incorporated. Cation mobility from limestone materials was negligible, and surface liming had no chemical effects in the solution collected $30 \mathrm{~cm}$ below soil surface.
\end{abstract}

Key words: surface liming, ion percolation, exchangeable cations

\section{INTRODUÇÃO}

Muitos solos catarinenses ainda são ácidos e apresentam teores de Al tóxicos às plantas (Ernani \& Almeida, 1986; Almeida et al., 1999) que limitam o aumento do rendimento vegetal (Ernani et al., 1998). Por isso, a calagem é uma prática agrícola normalmente aceita sem restrições pelos produtores, e que, na maioria das situações, proporciona benefícios econômicos (Pottker \& Ben, 1998; Oliveira et al., 1997).
Nos últimos anos, entretanto, com o aumento das áreas de cultivo sob o sistema de plantio direto, muitos produtores e técnicos estão questionando a necessidade da incorporação de calcário ao solo e até mesmo a eficiência da calagem, pois em algumas situações ela não tem aumentado o rendimento vegetal (Pottker \& Ben, 1998; Caires et al., 1999; Rheinheimer et al., 2000). Muitas lavouras atualmente sob plantio direto receberam grandes quantidades de fertilizantes fosfatados, e em condições de alta disponibilidade de $\mathrm{P}$, 
a resposta das culturas à calagem é baixa ou mesmo inexistente (Vidor \& Freire, 1972; Ernani et al., 1998; 2000), independentemente do sistema de preparo do solo (Ernani et al., 1999). A incorporação de calcário normalmente promove maiores rendimentos que a calagem superficial, principalmente nos primeiros anos (Weirich Neto et al., 2000), porém pouco se avançou nesse tópico nos últimos anos. Constatou-se que a calagem superficial promove incrementos no rendimento das culturas, cuja magnitude varia com a espécie e tipo de solo (Pottker \& Ben, 1998; Rheinheimer et al., 2000), condições de fertilidade, e principalmente com o $\mathrm{pH}$.

Os efeitos da calagem (elevação do $\mathrm{pH}$, do $\mathrm{Ca}$ e $\mathrm{Mg}$, e neutralização do Al trocável) normalmente restringem-se às zonas de aplicação ou imediatamente abaixo delas (Pottker \& Ben, 1998). Os valores de $\mathrm{pH}$ e $\mathrm{Al}$ alteram-se pouco em profundidade devido à baixa solubilidade dos corretivos agrícolas da acidez e à alta reatividade de seus ânions com os ácidos presentes na camada de solo em que o calcário é incorporado. A baixa mobilidade de $\mathrm{Ca}$ e $\mathrm{Mg}$, por outro lado, deve-se à pequena permanência dos ânions adicionados pelo calcário na solução do solo. Sendo assim, a quase totalidade do $\mathrm{Ca}$ e do $\mathrm{Mg}$ adicionada vai para as cargas negativas criadas pelo aumento do $\mathrm{pH}$, e para as cargas anteriormente ocupadas pelo $\mathrm{Al}$, e só uma pequena parte fica na solução do solo. A taxa de movimentação do $\mathrm{Ca}$ e do Mg no perfil depende, portanto, da existência de outros ânions na solução do solo, principalmente nitratos, cloretos e sulfatos, oriundos principalmente da mineralização da matéria orgânica, e cujas quantidades podem explicar, pelo menos em parte, a mobilidade diferencial dos efeitos do calcário em diferentes solos (Pearson et al., 1962; Gonzalez-Erico et al., 1979). Algumas características intrínsecas dos solos, principalmente aquelas relacionadas com 0 tamponamento, também afetam a profundidade de atuação dessas reações, que em alguns casos podem atingir camadas de até $40-60 \mathrm{~cm}$ de profundidade (Quaggio et al., 1982; Morelli et al., 1992).

$\mathrm{O}$ gesso agrícola $\left(\mathrm{CaSO}_{4} \cdot 2 \mathrm{H}_{2} \mathrm{O}\right)$, por outro lado, tem sido utilizado em solos ácidos como um produto complementar ao calcário, com o objetivo de diminuir a toxicidade do $\mathrm{Al}$ e aumentar a concentração de $\mathrm{Ca}$ em profundidade (Ritchey et al., 1980; Braga et al., 1995; Dias et al., 1994; Farina et al., 1988; Ernani, 1986; Ernani et al., 1993; Silva et al., 1998). A grande mobilidade vertical de cátions ocasionada pelo gesso (Ernani, 1986; Caires et al., 1998), deve-se a maior solubilidade desse produto em relação aos calcários, à inalteração das cargas elétricas, e à permanência do ânion sulfato quase que totalmente na solução do solo (Ernani, 1986; Ernani \& Barber, 1993; Dias et al., 1994).

O conhecimento da composição química da fase sólida e da água que percola no perfil pode fornecer subsídios para o entendimento do efeito benéfico ocasionado pela aplicação superficial de calcário e de gesso agrícola no desenvolvimento das plantas, e com esse objetivo conduziu-se o presente trabalho.

\section{MATERIAL E MÉTODOS}

O trabalho foi conduzido em laboratório, em 1997, no Centro de Ciências Agroveterinárias da Universidade do Estado de Santa Catarina (UDESC), em Lages (SC). Foram utilizados um Latossolo Bruno Álico (LB) e um Cambissolo Húmico Álico $(\mathrm{CH})$, coletados na camada de 0-20 cm de profundidade, em áreas de vegetação campestre nativa. Esses solos apresentavam, respectivamente, $\mathrm{pH}-\mathrm{CaCl}_{2}=4,05$ e 3,70, $\mathrm{pH}-\mathrm{H}_{2} \mathrm{O}=4,8$ e 4,45, Al ${ }^{3+}=2,4$ e $4,5 \mathrm{cmol}_{\mathrm{c}} \mathrm{kg}^{-1}, \mathrm{Ca}=1,2$ e $1,1 \mathrm{cmol}_{\mathrm{c}}$ $\mathrm{kg}^{-1}, \mathrm{Mg}=0,4$ e $0,7 \mathrm{cmol}_{\mathrm{c}} \mathrm{kg}^{-1}$, argila $=550$ e $410 \mathrm{mg} \mathrm{kg}^{-1}$, e matéria orgânica $=40$ e $60 \mathrm{~g} \mathrm{~kg}^{-1}$.

Os tratamentos consistiram de dois métodos de aplicação (com e sem incorporação aos solos ) e de três produtos agrícolas $\left(\mathrm{CaCO}_{3}, \mathrm{MgCO}_{3}\right.$, e gesso agrícola) em cada um dos solos, em experimentos independentes. $\mathrm{O}$ $\mathrm{CaCO}_{3}$ foi aplicado na dose equivalente a $25 \%$ da recomendada pelo método SMP $(0,25-\mathrm{SMP})$ para elevar o $\mathrm{pH}$ até 6,0 (3,5 e 4,5 tha ${ }^{-1}$, respectivamente no LB e no $\mathrm{CH}$ ); as quantidades de $\mathrm{MgCO}_{3}$ e de gesso agrícola foram calculadas de modo a aplicar a mesma quantidade de cátions que a adicionada pelo $\mathrm{CaCO}_{3}$ (respectivamente 2,91 e $9,24 \mathrm{t} \mathrm{ha}^{-1}$ no LB, e 3,8 e $12,1 \mathrm{t}^{3}$ ha $^{-1}$ no $\mathrm{CH}$ ). Com o objetivo de obter-se uma curva de neutralização da acidez, foram incorporadas aos solos doses adicionais de $\mathrm{CaCO}_{3}$, equivalentes a $0,0,50,1,0$ e 1,5 vez a recomendação do método SMP (respectivamente $0 ; 3,5 ; 7,0 ; 14$ e $21 \mathrm{tha}^{-1}$ no LB, e 0 ; 4,$5 ; 9,0 ; 18$ e $27 \mathrm{t} \mathrm{ha}^{-1}$ no $\mathrm{CH}$ ).

Utilizaram-se unidades experimentais de $1,5 \mathrm{~kg}$ de solo (base seca), e três repetições, distribuídas segundo o delineamento experimental completamente casualizado. Os tratamentos incorporados aos solos foram aplicados trinta dias antes que os demais, os quais foram adicionados quando as unidades experimentais foram transferidas para as colunas de lixiviação. Durante esses trinta dias de incubação, todas as unidades experimentais foram mantidas em sacos de polietileno, na temperatura ambiente, com umidade de aproximadamente $80 \%$ do teor de água retido na capacidade de campo.

As colunas de lixiviação foram construídas de $P V C$ rígido, e tinham $10 \mathrm{~cm}$ de diâmetro e $30 \mathrm{~cm}$ de altura. A parte inferior foi vedada com uma lâmina de isopor e fita adesiva, na qual fez-se um orifício central com $0,5 \mathrm{~cm}$ de diâmetro onde conectou-se uma mangueira plástica a fim de direcionar o fluxo da solução percolante. Acima da lâmina de isopor, colocou-se uma tela plástica para impedir a passagem de partículas de solo e evitar o entupimento do orifício de drenagem.

Uma semana após a transferência do solo para as colunas de lixiviação, iniciou-se a aplicação de água 
destilada sobre as mesmas. Este procedimento foi repetido a cada sete dias, durante doze semanas. $\mathrm{Na}$ primeira percolação, usou-se $400 \mathrm{~mL}$ de água por coluna; nas demais, $200 \mathrm{~mL}$, num tempo de aplicação de aproximadamente uma hora, totalizando $2600 \mathrm{~mL}$ por coluna durante todo o período experimental, quantidade essa equivalente a $300 \mathrm{~mm}$ de chuva.

A solução percolada foi coletada no dia seguinte à cada aplicação de água, e em cada uma delas determinou-se o volume, o pH, e as concentrações de $\mathrm{Ca}, \mathrm{Mg}$ e Al. O pH foi determinado por potenciometria, e os demais por espectrofotometria de emissão induzida por plasma (ICP).

Nos tratamentos incorporados, coletaram-se amostras de solo antes do início das percolações e duas semanas após o término das mesmas; nos tratamentos aplicados em superfície, coletaram-se amostras somente no final do experimento, nas profundidades de 0 a $2 \mathrm{~cm}$ e 2 a $30 \mathrm{~cm}$. Todas as amostras de solo foram secas em estufa com circulação de ar, a $60^{\circ} \mathrm{C}$, posteriormente moídas, e peneiradas em malha de $2 \mathrm{~mm}$. Nelas, determinaram-se os valores de $\mathrm{pH}, \mathrm{Ca}, \mathrm{Mg}, \mathrm{Al}$ e $\mathrm{K}$ trocáveis. $\mathrm{O} \mathrm{pH}$ foi determinado em água, na relação solo/solvente de $1: 1 ; \mathrm{Ca}, \mathrm{Mg}$, e $\mathrm{Al}$ foram extraídos com solução de $\mathrm{KCl} 1,0 \mathrm{~mol} \mathrm{~L}^{-1} \mathrm{e}$ determinados por espectrofotometria de emissão induzida por plasma.

A análise estatística do efeito dos corretivos e do gesso agrícola sobre as características químicas da fase sólida e da água percolada em cada solo consistiu na análise da variância segundo um delineamento inteiramente casualizado, com três repetições. Como praticamente não houve variação na composição química das soluções percoladas entre as diferentes épocas, o $\mathrm{pH}$ foi analisado usando-se a média, e os demais elementos usando-se o somatório de todas as percolações. A diferença entre médias de tratamentos foi avaliada pelo teste de Tukey a $5 \%$. As alterações nas características químicas da fase sólida e da água percolada em função da incorporação de níveis crescentes de $\mathrm{CaCO}_{3}$ foram avaliadas a partir da significância dos coeficientes de determinação de regressões polinomiais a $1 \%$ e $5 \%$.

\section{RESULTADOS E DISCUSSÃO}

\section{Efeitos no pH do solo}

$\mathrm{O} \mathrm{pH}$ da fase sólida e da solução percolada aumentou quadraticamente com o aumento da quantidade de $\mathrm{CaCO}_{3}$ incorporada (TABELA 1). Nos dois solos, a aplicação da quantidade de carbonato de cálcio necessária para elevar o pH até 6,0 (1,0 SMP) aumentou $\mathrm{o} \mathrm{pH}$ da solução percolada de aproximadamente 4,7 para 7,2 no Cambissolo Húmico (CH) e para 7,7 no Latossolo Bruno (LB) (TABELA 1); na fase sólida, o aumento foi até aproximadamente 6,5, nos dois solos (TABELA 2).
$\mathrm{O}$ pH da solução lixiviada foi sempre maior que o pH da fase sólida, nos dois solos, devido ao efeito de diluição, uma vez que um grande volume de água percolou pela coluna num curto espaço de tempo, simulando o que ocorre nos períodos subsequentes a chuvas prolongadas ou de altas intensidades. Nesse caso, não há tempo para a água que passa pelo solo estabelecer um novo equilíbrio com a fase sólida, e, por isso, a solução que percola é mais diluída que a que estava em equilíbrio com o solo antes do início da percolação. Devido a isso, durante os períodos de percolação de grandes quantidades de água, as plantas cultivadas em solos contendo $\mathrm{Al}$, devem beneficiar-se desse aumento temporário de $\mathrm{pH}$, pois estarão absorvendo uma solução cuja atividade do Al é menor que a verificada em períodos sem excesso de precipitação.

$\mathrm{O} \mathrm{CaCO}_{3}$, na dose de $3,5 \mathrm{t} \mathrm{ha}^{-1}$ no LB e de $4,5 \mathrm{t} \mathrm{ha}^{-1}$ no $\mathrm{CH}(0,25-\mathrm{SMP})$, ou a dose molar equivalente de $\mathrm{MgCO}_{3}\left(2,91\right.$ e 3,81 $\mathrm{t} \mathrm{ha}^{-1}$ respectivamente em cada solo), sobre a superfície do solo, não afetaram o pH da solução lixiviada. Quando essas quantidades foram incorporadas, o $\mathrm{pH}$ aumentou de 4,6 para 5,9 no LB e de 4,7 para 5,8 no $\mathrm{CH}$ (TABELAS 1 e 2), e mostra que, nos tratamentos aplicados sobre a superfície, o pH da solução diminuiu à medida que ela percolou nos solos.

A aplicação superficial desses corretivos elevou o $\mathrm{pH}$ somente da camada de $0-2 \mathrm{~cm}$, para próximo de 7,0 (TABELA 3), e demonstra a pequena reatividade do calcário abaixo dos locais de aplicação, à semelhança do encontrado por Pottker \& Ben (1998) e por Rheinheimer et al. (2000). Alguns autores têm observado elevação do $\mathrm{pH}$ em camadas bem abaixo daquelas onde o calcário tem sido aplicado (Quaggio et al., 1982; Morelli et al., 1992), e essas diferenças estão, provavelmente, relacionadas ao tamponamento dos solos.

A adição de gesso agrícola, independente do modo de aplicação, diminuiu o pH da solução percolada (TABELA 1) e da fase sólida (TABELA 2) em aproximadamente quatro e duas unidades decimais respectivamente. Essa diminuição de $\mathrm{pH}$, diferentemente do encontrado por outros autores (Ernani, 1986; Caires et al., 1998), foi, provavelmente, ocasionada pela hidrólise do $\mathrm{Al}$, deslocado das cargas negativas pelo $\mathrm{Ca}$ aplicado (Ernani \& Barber, 1993), e também pelo aumento da concentração de eletrólitos, uma vez que esses dois solos têm predomínio de carga líquida negativa.

\section{Efeitos no Cálcio}

Tanto o Ca trocável quanto o Ca percolado aumentaram com a dose de $\mathrm{CaCO}_{3}$ incorporada, nos dois solos (TABELAS 1, 2 e 4). Relativamente à testemunha, a incorporação da quantidade equivalente a 1,0-SMP (18 $\mathrm{t} \mathrm{ha}^{-1}$ no $\mathrm{CH}$ e $14 \mathrm{t} \mathrm{ha}^{-1}$ no LB) percolou sete vezes mais Ca no $\mathrm{LB}$ e 4,5 vezes mais no $\mathrm{CH}$, porém isso equivaleu a uma perda respectivamente de apenas 4,8 e 2,5\% do $\mathrm{Ca}$ aplicado. Esse tratamento aumentou a concentração 
média de Ca no lixiviado de 18 para $81 \mathrm{mg} \mathrm{L}^{-1}$ no $\mathrm{CH}$, e de 16 para $112 \mathrm{mg} \mathrm{L}^{-1}$ no LB (TABELA 1) e o Ca trocável, respectivamente, em 110 e $80 \mathrm{mmol}_{\mathrm{c}} \mathrm{kg}^{-1}$ (TABELA 2). Soprano \& Alvarez (1989) também encontraram perdas de $\mathrm{Ca}$ inferiores a $10 \%$ do aplicado na forma de carbonato.

Diferentemente da incorporação, a aplicação dos corretivos sobre a superfície, na dose 0,25 SMP, não afetou o Ca percolado em nenhum solo (TABELA 1), e mostra que o Ca reagiu com o solo à medida que desceu na coluna. $\mathrm{Na}$ fase sólida, a aplicação superficial do $\mathrm{CaCO}_{3}$ elevou o $\mathrm{Ca}$ trocável, na camada de 0 a 2,0 cm, em aproximadamente $150 \mathrm{mmol}_{\mathrm{c}} \mathrm{kg}^{-1}$ no $\mathrm{CH}$ e em 110 mmol ${ }_{\mathrm{c}} \mathrm{kg}^{-1}$ no LB; na camada de 2,1 a $30 \mathrm{~cm}$ de profundidade, o Ca trocável, entretanto, não foi afetado no $\mathrm{CH}$ e aumentou apenas $10 \mathrm{mmol}_{\mathrm{c}} \mathrm{kg}^{-1}$ no LB (TABELA 3). Essa é a dose recomendada nos Estados do Rio Grande do Sul e Santa Catarina para situações em que o calcário não é incorporado com o solo.

TABELA 1 - Valores de pH, Ca, Mg e Al lixiviados de colunas com $30 \mathrm{~cm}$ de profundidade em função da incorporação (Inc) ou da aplicação superficial (Sup) de carbonato de magnésio( ${ }^{(1)}$, de gesso agrícola ${ }^{(1)}$ e de doses crescentes de carbonato de cálcio $^{(2)}$ em dois solos ácidos catarinenses. Média de duas repetições.

\begin{tabular}{|c|c|c|c|c|c|c|c|c|}
\hline \multirow{2}{*}{ Tratame nto } & \multicolumn{4}{|c|}{ Cambissolo Húmico } & \multicolumn{4}{|c|}{ Latossolo Bruno } \\
\hline & $\mathrm{pH}$ & $\mathrm{Ca}$ & $\mathrm{Mg}$ & $\mathrm{Al}$ & $\mathrm{pH}$ & $\mathrm{Ca}$ & $\mathrm{Mg}$ & $\mathrm{Al}$ \\
\hline & \multicolumn{4}{|c|}{--------- mg coluna-1 --------- } & \multicolumn{4}{|c|}{-------------- mg coluna'-1 ------------ } \\
\hline Testemunha & $4,7 \mathrm{f}$ & $30,2 \mathrm{e}$ & 13,9 def & $2,0 \mathrm{c}$ & $4,6 \mathrm{e}$ & $28,1 \mathrm{f}$ & $6,7 \mathrm{c}$ & $1,24 \mathrm{e}$ \\
\hline $0,25 \mathrm{CaCO}_{3}$ Inc & $5,8 \mathrm{~d}$ & 70,0 de & 8,2 def & $2,3 \mathrm{c}$ & $5,9 d$ & $47,7 \mathrm{f}$ & $3,3 \mathrm{c}$ & $3,2 \mathrm{~d}$ \\
\hline $0,25 \mathrm{CaCO}_{3}$ Sup & $5,4 \mathrm{e}$ & $40,0 \mathrm{e}$ & $17,7 \mathrm{f}$ & $2,1 \mathrm{c}$ & $4,8 \mathrm{e}$ & $41,2 \mathrm{f}$ & $6,2 \mathrm{c}$ & $1,2 \mathrm{e}$ \\
\hline $0,5 \mathrm{CaCO}_{3} \mathrm{Inc}$ & $6,6 \mathrm{c}$ & $97,8 \mathrm{~cd}$ & 6,2 ef & $4,1 \mathrm{c}$ & $6,6 \mathrm{c}$ & $88,5 \mathrm{e}$ & $4,2 \mathrm{c}$ & $3,9 \mathrm{~b}$ \\
\hline $1,0 \mathrm{CaCO}_{3}$ Inc & $7,2 \mathrm{~b}$ & $137 \mathrm{c}$ & $4,7 \mathrm{f}$ & $1,9 \mathrm{c}$ & $7,7 \mathrm{~b}$ & $201 \mathrm{c}$ & $5,0 \mathrm{c}$ & $2,8 \mathrm{~cd}$ \\
\hline $1,5 \mathrm{CaCO}_{3}$ Inc & $8,0 \mathrm{a}$ & $230 \mathrm{~b}$ & $5,2 \mathrm{f}$ & $2,1 \mathrm{c}$ & $8,1 \mathrm{a}$ & $281 \mathrm{~b}$ & $4,3 \mathrm{c}$ & $2,3 d$ \\
\hline $\mathrm{MgCO}_{3} \mathrm{Inc}$ & $5,8 d$ & $18,8 \mathrm{e}$ & $57,3 \mathrm{c}$ & $2,5 \mathrm{c}$ & $5,7 d$ & $9,9 \mathrm{f}$ & $25 a$ & $2,3 d$ \\
\hline $\mathrm{MgCO}_{3}$ Sup & $4,8 \mathrm{f}$ & $41,2 \mathrm{e}$ & $18,9 d$ & $2,4 \mathrm{c}$ & $4,7 \mathrm{e}$ & $25,9 \mathrm{f}$ & $7,0 \mathrm{c}$ & $1,2 \mathrm{e}$ \\
\hline Gesso Inc & $4,4 \mathrm{~g}$ & 980 a & $83,2 \mathrm{a}$ & $77,2 \mathrm{a}$ & $4,2 \mathrm{f}$ & 54,9 a & $24,9 a$ & 13,6 a \\
\hline Gesso Sup & $4,3 \mathrm{~g}$ & $251 \mathrm{~b}$ & $69,3 \mathrm{~b}$ & $22,7 \mathrm{~b}$ & $4,3 \mathrm{f}$ & $14,2 \mathrm{~d}$ & $20,2 \mathrm{~b}$ & $4,5 \mathrm{bc}$ \\
\hline
\end{tabular}

(1)A dose aplicada supriu a mesma quantidade de cátion que a dose 0,25 de $\mathrm{CaCO}_{3}$. ${ }^{(2)}$ Fração da dose integral recomendada pelo método SMP para elevar o pH dos solos ate 6.0. Letras iguais, dentro da coluna, num mesmo parâmetro químico, significa ausência de diferença estatística entre a média dos tratamentos. Dividindo-se a quantidade percolada de cada elemento (mg coluna-1) por 1,8 no LB e por 1,7 no $\mathrm{CH}$, encontra-se a concentração de cada elemento $\left(\mathrm{mg} \mathrm{L}^{-1}\right)$ em cada percolação, a qual praticamente não variou entre as lixiviações.

TABELA 2 - Valores de pH, Ca, Mg e Al trocáveis, antes e após as percolações, em função da aplicação de carbonato de magnésio, gesso agrícola, e de quantidades crescentes de carbonato de cálcio incorporados a dois solos ácidos catarinenses. Média de duas repetições.

\begin{tabular}{|c|c|c|c|c|c|c|c|c|}
\hline \multirow{2}{*}{ Tratamento } & \multicolumn{2}{|c|}{$\mathrm{pH}$} & \multicolumn{2}{|c|}{$\mathrm{Ca}$} & \multicolumn{2}{|c|}{$\mathrm{Mg}$} & \multicolumn{2}{|c|}{$\mathrm{Al}$} \\
\hline & Antes & Depois & Antes & Depois & Antes & Depois & Antes & Depois \\
\hline & \multicolumn{8}{|c|}{ - - } \\
\hline & \multicolumn{8}{|c|}{ Latossolo Bruno } \\
\hline Testemunha & 4,3 a $E$ & $4,4 \mathrm{a}$ & 12 a $E$ & $11 \mathrm{a}$ & $4 \mathrm{~B}$ & 3 & $25 \mathrm{~A}$ & 2,6 \\
\hline $0,25 \mathrm{CaCO}_{3}$ & 4,9 a D & $5,0 \mathrm{a}$ & 38 a D & $35 a$ & $3 \mathrm{~B}$ & 3 & $06 \mathrm{~B}$ & 7 \\
\hline $0,50 \mathrm{CaCO}_{3}$ & 5,6 a C & $5,5 \mathrm{a}$ & 65 a C & $55 \mathrm{~b}$ & $3 \mathrm{~B}$ & 3 & $0,0 \mathrm{C}$ & 0,0 \\
\hline $1,0 \mathrm{CaCO}_{3}$ & 6,6 a $B$ & $6,3 a$ & 91 a B & $84 \mathrm{~b}$ & $4 \mathrm{~B}$ & 4 & $0,0 \mathrm{C}$ & 0,0 \\
\hline $1,5 \mathrm{CaCO}_{3}$ & 7,1 a $A$ & $6,9 a$ & 103 a $A$ & $97 \mathrm{~b}$ & $3 \mathrm{~B}$ & 3 & $0,0 \mathrm{C}$ & 0,0 \\
\hline $0,25 \mathrm{MgCO}_{3}$ & 5,0 a D & $5,0 \mathrm{a}$ & 11 a $E$ & $10 \mathrm{~b}$ & $32 \mathrm{~A}$ & 30 & $8 \mathrm{~B}$ & 8 \\
\hline \multirow[t]{2}{*}{$0,25 \mathrm{CaSO}_{4}$} & 4,2 a $E$ & $4,3 a$ & 62 a D & $35 a$ & $6 \mathrm{~B}$ & 4 & $23 \mathrm{~A}$ & 24 \\
\hline & \multicolumn{8}{|c|}{ Cambissolo Húmico } \\
\hline Testemunha & 4,5 a $E$ & $4,0 \mathrm{a}$ & 12 a $\mathrm{E}$ & $11 \mathrm{a}$ & 7 a B & $6 a$ & $44 \mathrm{~A}$ & 47 \\
\hline $0,25 \mathrm{CaCO}_{3}$ & 5,0 a D & $5,0 \mathrm{a}$ & 48 a D & $45 \mathrm{a}$ & 7 a B & $7 a$ & $16 \mathrm{~B}$ & 16 \\
\hline $0,50 \mathrm{CaCO}_{3}$ & 5,7 a C & $5,6 a$ & 90 a $C$ & $79 b$ & 6 a B & $6 a$ & $0,0 \mathrm{C}$ & 0,0 \\
\hline $1,0 \mathrm{CaCO}_{3}$ & 6,5 a $\mathrm{B}$ & $6,2 \mathrm{~b}$ & 117 a B & $108 b$ & 4 a BC & $4 a$ & $0,0 \mathrm{C}$ & 0,0 \\
\hline $1,5 \mathrm{CaCO}_{3}$ & 6,8 a $A$ & $6,7 \mathrm{a}$ & 139 a $A$ & $131 \mathrm{~b}$ & 3 a BC & $3 a$ & $0,0 \mathrm{C}$ & 0,0 \\
\hline $0,25 \mathrm{MgCO}_{3}$ & 4,9 a D & $5,0 \mathrm{a}$ & 13 a $E$ & $12 b$ & 46 a $A$ & $41 \mathrm{~b}$ & $18 \mathrm{~A}$ & 19 \\
\hline $0,25 \mathrm{CaSO}_{4}$ & 4,2 a F & $4,3 \mathrm{a}$ & 81 a D & $42 \mathrm{a}$ & 9 a BC & $5 \mathrm{~b}$ & $46 \mathrm{~A}$ & 40 \\
\hline
\end{tabular}

Letras minúsculas iguais, na linha, dentro de um mesmo parâmetro, significa ausência de diferença estatística entre antes e depois, a 5\%; letras maiúsculas iguais, dentro da coluna, num mesmo parâmetro químico, significa ausência de diferença estatística entre a média dos tratamentos químicos. 
Mobilidade de $\mathrm{Ca}$ e de $\mathrm{Mg}$ oriundos da incorporação de calcário na camada arável tem sido observado em alguns solos até profundidades superiores a $40 \mathrm{~cm}$ (Quaggio et al., 1982; Morelli et al., 1992), e essas diferenças provavelmente se devem ao número de cargas elétricas negativas integrantes da CTC, e ao teor de matéria orgânica, que além de afetar as cargas, afeta a quantidade de ânions presentes na solução do solo.

O gesso agrícola percolou bastante $\mathrm{Ca}$, principalmente quando incorporado aos solos (TABELA 1). Relativamente à testemunha, a quantidade de $\mathrm{Ca}$ percolada aumentou 32 vezes no $\mathrm{CH}$, quando o gesso foi incorporado, e oito vezes quando ele foi deixado sobre a superfície; no LB, os aumentos foram de 20 e 5 vezes respectivamente (TABELA 1). No tratamento incorporado, isso representa uma perda de 65 e $48 \%$ do $\mathrm{Ca}$ adicionado em cada solo. Esses valores são muito maiores que os observados por Soprano \& Alvarez (1989), onde as perdas atingiram somente $12,6 \%$ do $\mathrm{Ca}$ adicionado na forma de gesso. No $\mathrm{CH}$, a concentração média de Ca na solução lixiviada passou de $18 \mathrm{mg} \mathrm{L}^{-1}$, na testemunha, para 148 e $576 \mathrm{mg} \mathrm{L}^{-1}$ respectivamente

TABELA 3 - Valores de $\mathrm{pH}, \mathrm{Ca}, \mathrm{Mg}$, e Al nas camadas de 0 $2 \mathrm{~cm}$ e de $2-30 \mathrm{~cm}$ de profundidade em dois solos ácidos em função da aplicação superficial de 0,25 da dose de $\mathrm{CaCO}_{3}$ necessária para elevar $\mathrm{o} \mathrm{pH}$ a 6,0 e de quantidades molares equivalentes nas formas de $\mathrm{MgCO}_{3}$ e gesso agrícola. Média de três repetições.

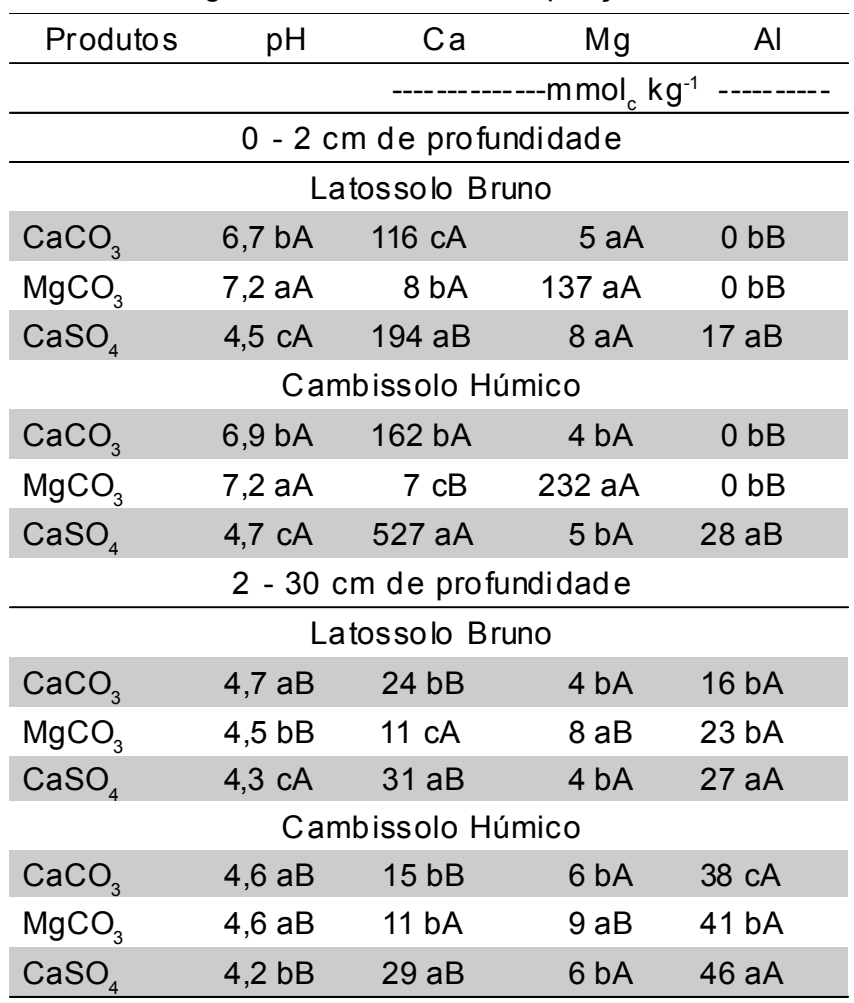

Médias seguidas da mesma letra em cada parâmetro químico (minúsculas entre os produtos, dentro de cada solo e profundidade, e maiúsculas entre as profundidades, dentro de cada produto e solo) não diferem estatisticamente pelo teste de Tukey a $5 \%$. pela aplicação de gesso na superfície ou incorporado; no LB, ela passou de $16 \mathrm{mg} \mathrm{L}^{-1}$ para respectivamente 79 e $305 \mathrm{mg} \mathrm{L}^{-1}$.

A menor lixiviação de Ca pelo gesso aplicado na superfície do solo do que pelo incorporado, foi devido a menor solubilização do gesso na superfície do solo, uma vez que as partículas entram em contato com menor volume de água. Ernani \& Barber (1993) mostraram que a mobilidade vertical de íons no solo, decorrente da aplicação de sulfato de cálcio, atinge um limite máximo, determinado pela limitação na solubilidade do produto.

$A$ adição de 2,6 $L$ de água por coluna, durante as doze semanas, que resultou numa percolação de 1,8 $\mathrm{L}$ no $\mathrm{LB}$ e de $1,7 \mathrm{~L}$ no $\mathrm{CH}$, diminuiu muito pouco o $\mathrm{Ca}$ trocável nos tratamentos que receberam $\mathrm{CaCO}_{3}$. Na dose de 1,0 SMP, o Ca trocável diminuiu de 117 para 108 $\mathrm{mmol}_{\mathrm{c}} \mathrm{kg}^{-1}$ no $\mathrm{CH}$ e de 91 para $84 \mathrm{mmol}_{\mathrm{c}} \mathrm{kg}^{-1}$ no LB (TABÉLA 2). A incorporação de gesso, entretanto, diminuiu o Ca trocável de 81 para $42 \mathrm{mmol}_{\mathrm{C}} \mathrm{kg}^{-1}$ no $\mathrm{CH}$ e de 62 para $35 \mathrm{mmol}_{\mathrm{c}} \mathrm{kg}^{-1}$ no LB (TABELA ${ }^{\mathrm{C}}$ 2). O maior efeito do gesso em movimentar Ca no solo, relativamente ao $\mathrm{CaCO}_{3}$, se deve à sua maior solubilidade, e à permanência do ânion sulfato na solução do solo (Ernani \& Barber, 1993; Dias et al., 1994), o qual é pouco adsorvido pela fase sólida principalmente em valores de pH superiores a 4,5 (Ernani, 1986). Quando bases fortes, como os calcários agrícolas, são aplicadas a solos ácidos, os ânions adicionados praticamente não ficam na solução, pois reagem com os ácidos do solo elevando o $\mathrm{pH}$.

Para culturas exigentes em $\mathrm{Ca}$, como amendoim e macieira, a aplicação de gesso ao solo é uma boa alternativa, principalmente em solos com alta CTC, onde a lixiviação de $\mathrm{Mg}$ e K não é tão grande (Ernani, 1986;

TABELA 4 - Equações de regressão ajustadas para $\mathrm{pH}, \mathrm{Ca}$, e Mg (trocáveis e na solução percolada) como variaveis dependentes $(\mathrm{y})$ das quantidades crescentes de carbonato de cálcio incorporadas a dois solos ácidos catarinenses.

\begin{tabular}{lcc}
\hline \multicolumn{1}{c}{ Parâmetro } & Equação de regressão & $\mathrm{r}^{2}$ \\
\hline & Latossolo Bruno & \\
$\mathrm{pH}$-solução & $\mathrm{y}=4,67+0,34 \mathrm{x}-8,4 \mathrm{x}^{2}$ & 0,99 \\
$\mathrm{pH}$ solo & $\mathrm{y}=4,25+0,22 \mathrm{x}-3,98 \mathrm{x}^{2}$ & 0,99 \\
Ca-percolado (mg) & $\mathrm{y}=160+153 \mathrm{x}$ & 0,99 \\
Ca-trocável(mmolc kg $\left.{ }^{-1}\right)$ & $\mathrm{y}=11,8+8,7 \mathrm{x}-0,21 \mathrm{x}^{2}$ & 0,99 \\
Mg-percolado & $\mathrm{y}=\mathrm{NS}$ & \\
\hline
\end{tabular}

Cambissolo Húmico

\begin{tabular}{lll} 
pH-solução & $y=4,82+0,208 x-3,48 x^{2}$ & 0,98 \\
pH solo & $y=4,44+0,161 x-2,69 x^{2}$ & 0,99 \\
Ca-percolado $(\mathrm{mg})$ & $y=380+84 x$ & 0,98 \\
Ca-trocável(mmolc kg-1) & $y=12,9+9,2 x-0,17 x^{2}$ & 0,99 \\
Mg-percolado & $y=158-11,9 x+0,31 x^{2}$ & 0,95 \\
\hline
\end{tabular}

$\mathrm{x}=\mathrm{tha}^{-1}$ de $\mathrm{CaCO}_{3}$. 
Ernani et al., 1993). Se aplicado sobre a superfície do solo, a magnitude da lixiviação será ainda menor, e o efeito residual será maior do que se o produto for incorporado ao solo.

\section{Efeitos no Magnésio}

A quantidade de $\mathrm{Mg}$ na solução percolada somente foi afetada pela incorporação de $\mathrm{CaCO}_{3}$ no $\mathrm{CH}$ (TABELA 4). A diminuição na lixiviação de $\mathrm{Mg}$ deveu-se, provavelmente, à sua diminuição na solução do solo, ocasionada pelo aumento das cargas dependentes de $\mathrm{pH}$ e pela adsorção do nutriente através de complexos de esfera interna. A aplicação de $\mathrm{MgCO}_{3}$, por outro lado, aumentou a quantidade de $\mathrm{Mg}$ percolada somente no tratamento incorporado. Nos dois solos, o Mg percolado aumentou aproximadamente quatro vezes relativamente à testemunha (TABELA 1), e passou de 8 para $34 \mathrm{mg} \mathrm{L}^{-1}$ no $\mathrm{CH}$ e de 4 para $14 \mathrm{mg} \mathrm{L}^{-1}$ no LB.

O gesso agrícola promoveu grande lixiviação de $\mathrm{Mg}$, à semelhança do encontrado por outros autores (Ernani, 1986; Ernani \& Barber, 1993; Silva et al., 1998), e a magnitude foi maior quando o produto foi incorporado do que quando aplicado na superfície (TABELAS 1 e 2 ). $\mathrm{A}$ incorporação de gesso percolou tanto ou mais $\mathrm{Mg}$ do que o tratamento onde incorporou-se $\mathrm{MgCO}_{3}$ (TABELA 1). No $\mathrm{CH}$, o gesso aplicado na superfície também percolou mais $\mathrm{Mg}$ do que o tratamento que recebeu $\mathrm{MgCO}_{3}$ incorporado (TABELA 1).

O Mg trocável somente aumentou pela aplicação dos tratamentos com $\mathrm{MgCO}_{3}$. As percolações, por outro lado, lixiviaram $\mathrm{Mg}$ do solo somente nos tratamentos que receberam gesso agrícola ou $\mathrm{MgCO}_{3}$ incorporados, numa magnitude de 4 e $5 \mathrm{mmol}_{\mathrm{c}} \mathrm{kg}^{-1}$, respectivamente.

\section{Efeitos no Alumínio}

A quantidade de Al percolada foi pequena e somente foi afetada pela aplicação de gesso agrícola (TABELA 1). Ausência de percolação de Al pela aplicação de $\mathrm{CaCO}_{3}$ também foi verificada por Amaral et al. (1998). Quando os corretivos da acidez são incorporados ao solo, o pH aumenta e o Al precipita na forma de $\mathrm{Al}(\mathrm{OH})_{3}$, saindo portanto da fase líquida do solo. Com a aplicação de gesso, parte do Al é deslocado pelo Ca aplicado (Ernani \& Barber, 1993), e pode ser lixiviado. Normalmente a quantidade de Al lixiviada não é muito grande porque o Al existe em quantidades muito pequenas na solução do solo (Pavan et al., 1982; Ernani \& Barber, 1993).

A partir da aplicação de quantidades iguais ou superiores a 0,5 vezes a quantidade de $\mathrm{CaCO}_{3}$ necessária para elevar o $\mathrm{pH}$ até 6,0 (0,5-SMP), o $\mathrm{Al}^{3}$ trocável deixou de existir nos dois solos (TABELA 2), independente do tipo de corretivo $\left(\mathrm{CaCO}_{3}\right.$ ou $\left.\mathrm{MgCO}_{3}\right)$, semelhantemente ao observado por Ernani et al. (1998), num solo semelhante de Santa Catarina. A aplicação de gesso não afetou o Al trocável em qualquer dos solos, diferentemente do encontrado por outros pesquisadores, que mostram que adições de grandes quantidades de gesso promovem pequeno decréscimo nessa forma de Al (Ernani, 1986; Nogueira \& Mozeto, 1990).

\section{CONCLUSÕES}

A incorporação dos corretivos da acidez aos solos aumentou o pH e a concentração do cátion acompanhante na solução percolada, porém a aplicação sobre a superfície não afetou nenhum parâmetro químico da fase liquida, e alterou a composição química da fase sólida somente na camada superficial de $0-2 \mathrm{~cm}$;

O gesso agrícola promoveu maior percolação de cátions do que os corretivos da acidez, principalmente quando incorporado aos solos. A aplicação do gesso na superfície do solo, diminui a magnitude da lixiviação e prolonga seu efeito residual.

\section{REFERÊNCIAS BIBLIOGRÁFICAS}

ALMEIDA, J.A.; ERNANI, P.R.; MAÇANEIRO, K.C. Recomendação alternativa de calcário para solos altamente tamponados do extremo sul do Brasil. Ciência Rural, v.29, p.651-656, 1999.

AMARAL, A.S.; ANGHINONI, I.; KRAY, C.H. Alteração de atributos químicos das fases sólidas e líquidas do solo pela reaplicação superficial de calcário no sistema de plantio direto consolidado. In: REUNIÃO SUL-BRASILEIRA DE CIÊNCIA DO SOLO, 2., Santa Maria, 1998. Resumos expandidos. Santa Maria: NRS-SBCS, 1998. p.150-153.

BRAGA, F.A.; VALE, F.R.; MUNIZ, J.A. Movimentação de nutrientes no solo, crescimento e nutrição mineral do eucalipto, em função de doses de gesso e níveis de irrigação. Revista Brasileira de Ciência do Solo, v.19, p.69-77, 1995.

CAIRES, E.F.; CHUEIRI, W.A.; MADRUGA E.F.; FIGUEIREDO, A. Alterações de características químicas do solo e resposta da soja ao calcário e gesso aplicados na superfície em sistema de cultivo sem preparo do solo. Revista Brasileira de Ciência do Solo, v.22, p.27-34, 1998.

CAIRES, E.F.; FONSECA, A.F.; MENDES, J.; CHUEIRI, W.A.; MADRUGA, E.F. Produção de milho, trigo e soja em função das alterações das características químicas do solo pela aplicação de calcário e gesso na superfície, em sistema plantio direto. Revista Brasileira de Ciência do Solo, v.23, p.315-327, 1999.

DIAS, L.E.; ALVAREZ, V.H.; COSTA, L.M.; NOVAIS, R.F. Dinâmica de algumas formas de enxofre em colunas de solos tratados com diferentes doses de fósforo e gesso. Revista Brasileira de Ciência do Solo, v.18, p.373-380, 1994.

ERNANI, P.R. Alterações em algumas características químicas na camada arável do solo pela aplicação de gesso agrícola sobre a superfície de campos nativos. Revista Brasileira de Ciência do Solo, v.10, p.241-245, 1986.

ERNANI, P.R.; ALMEIDA, J.A. Comparação de métodos analíticos para avaliar a necessidade de calcário dos solos do Estado de Santa Catarina. Revista Brasileira de Ciência do Solo, v.10, p.143-150, 1986.

ERNANI, P.R.; BARBER S.A. Composição da solução do solo e lixiviação de cátions afetadas pela aplicação de cloreto e sulfato de cálcio em um solo ácido. Revista Brasileira de Ciência do Solo, v.10, p.41-46, 1993.

ERNANI, P.R.; CASSOL, P.C.; MORIGUTII, H.; GARCIA, M.M.; VACARO, M. Aplicação de gesso agrícola e lixiviação de potássio em solos catarinenses. Universidade \& Desenvolvimento, v.1, p.7-16, 1993. 
ERNANI, P.R.; NASCIMENTO, J.A.; OLIVEIRA L.C. Aumento do rendimento de grãos e de massa verde de milho ocasionado pela calagem. Revista Brasileira de Ciência do Solo, v.22, p.275-280, 1998.

ERNANI, P.R.; NASCIMENTO, J.A.L.; CAMPOS, M.L.; CAMILLO, R.J. Influência da combinação de fósforo e calcário no rendimento de milho. Revista Brasileira de Ciência do Solo, v.24, p.537-544, 2000.

ERNANI, P.R.; BAYER, C.; MAESTRI, L. Influência da calagem e do sistema de preparo do solo no rendimento de milho. In: REUNIÃO TÉCNICA CATARINENSE DE MILHO E FEIJÃO, 2., Lages, 1999. Resumos expandidos. Lages: UDESC, 1999. p.181-183.

FARINA, M.P.W.; CHANNON, P. Acid-subsoil amelioration: II. Gypsum effects on growth and subsoil chemical properties. Soil Science Society of America Journal, v.52, p.175-180, 1988.

GONZALEZ-ERICO E.; KAMPRATH, E.J.; NADERMAN, G.C.; SOARES, W.V. Effect of depth of lime incorporation on growth of corn on an Oxisol of Central Brazil. Soil Science Society of America Journal, v.43, p.1155-1158, 1979.

MORELLI, J.L.; DALBEN, A.E.; ALMEIDA, J.O.C.; DEMATTÊ, J.L.I. Calcário e gesso na produtividade da cana-de-açúcar e nas características químicas de um latossolo de textura médio alíco. Revista Brasileira de Ciência do Solo, v.16, p.187194, 1992

NOGUEIRA, A.R.A.; MOZETO, A.A. Interações químicas do sulfato e carbonato de cálcio em seis solos paulistas sob vegetação de cerrado. Revista Brasileira de Ciência do Solo, v.14, p.1-6, 1990.

OLIVEIRA, E.L.; PARRA, M.S.; COSTA, A. Resposta da cultura do milho, em latossolo vermelho-escuro Álico, à calagem. Revista Brasileira de Ciência do Solo, v.21, p.65-70, 1997.

PAVAN, M.A; BINGHAM, F.T.; PRATT, P.F. Toxicity of aluminum to coffee in Ultisols and Oxisols amended with $\mathrm{CaCO}_{3}, \mathrm{MgCO}_{3}$ and $\mathrm{CaSO}_{4} \cdot 2 \mathrm{H}_{2} \mathrm{O}$. Soil Science Society of America Journal, v.46, p.1201-1207, 1982.
PEARSON, R.W.; ABRUNA, F.; VICENT-CHANDLER, J.E. Effect of lime and nitrogen applications on downard movement of calcium and magnesium in two humid tropical soil of Puerto Rico. Soil Science, v.93, p.77-82, 1962.

POTTKER, D.; BEN, J.R. Calagem para uma rotação de culturas no sistema plantio direto. Revista Brasileira de Ciência do Solo, v.22, p.75-684, 1998.

QUAGGIO, J.A.; MASCARENHAS, H.A.A.; BATAGLIA, O.C. Resposta da soja a aplicação de doses crescentes de calcário em Latossolo roxo distrófico de cerrado. Revista Brasileira de Ciência do Solo, v.6, p.113-118, 1982.

RHEINHEIMER, D.S.; SANTOS, E.J.S.; KAMINSKI, J.; XAVIER, F.M. Aplicação superficial de calcário no sistema plantio direto consolidado em solo arenoso. Ciência Rural, v.30, p.263268, 2000

RITCHEY, K.D.; SOUZA, D.M.G.; LOBATO, E.; CORREA, O. Calcium leaching to increase rooting depth in Brazilian savannanh oxisol. Agronomy Journal, v.72, p.40-44, 1980.

SILVA, A.A.; VALE, F.R.; FERNANDES, L.A.; FURTINI-NETO, A.E.; MUNIZ, J.A. Efeitos de relações $\mathrm{CaSO}_{4} / \mathrm{CaCO}_{3}$ na mobilidade de nutrientes no solo e no crescimento do algodoeiro. Revista Brasileira de Ciência do Solo, v.22, p.451-457, 1998.

SOPRANO, E.; ALVAREZ, V.H. Nutrientes lixiviados de colunas de solo tratadas com diferentes sais de cálcio. Revista Brasileira de Ciência do Solo, v.13, p.25-29, 1989.

VIDOR, C.; FREIRE, J.R.J. Efeito da calagem e da adubação fosfatada sobre a fixação simbiótica do nitrogênio pela soja (Glycine max (L.) Merrill). Agronomia Sulriograndense, v.7, p.181-190, 1972.

WEIRICH NETO, P.H.; CAIRES, E.F.; JUSTINO, A.; DIAS, J. Correção da acidez do solo em função de modos de incorporação de calcário. Ciência Rural, v.30, p.257-261, 2000.

$\overline{\text { Recebido em } 17.07 .00}$ 